\title{
Correction of Cardio-hemodynamics and Endothelial Function Disorders in Pregnant Women with Connective Tissue Dysplasia
}

\section{Viktoria Yuriivna Dobrianska ${ }^{1}$, Svitlana Mykolaivna Heryak ${ }^{2 *}$ and Mykola Ivanovych Shved ${ }^{3}$}

${ }^{1}$ Department of Obstetrics and Gynaecology No.1, I. Horbachevsky Ternopil

National Medical University, Ukraine

${ }^{2}$ Department of Obstetrics and Gynaecology No. 2, I. Horbachevsky Ternopil

National Medical University, Ukraine

${ }^{3}$ Department of First Emergency Medical Aid and Emergency Medical Treatment I.

Horbachevsky Ternopil National Medical University, Ukraine

*Corresponding Author: Svitlana Mykolaivna Heryak, Department of Obstetrics and Gynaecology No. 2, I. Horbachevsky Ternopil National Medical University,

Ukraine.
Received: January 03, 2022

Published: January 27, 2022

(C) All rights are reserved by Svitlana

Mykolaivna Heryak, et al.

\begin{abstract}
Objectives: The negative impact of undifferentiated connective tissue dysplasia (UCTD) in myocardial contractility and endothelial microvascular dysfunction and their frequent combination with cardiac arrhythmias suggests an unfavorable course to pregnancy and childbirth due to the formation of uteroplacental dysfunction.

The Aim of the Study: To reduce the incidence of obstetric complications in pregnants with UCTD by carnitine-arginine complex. Materials and Methods: There were examined 58 pregnants with UCTD, including mitral valve prolaps with and without frequent extrasystoles, and 14 pregnants of control group. Metabolic support by intravenous infusion of L-arginine and L-carnitine complex.

Results: There are violations of morpho-functional parameters of the heart with the development of moderate systolic-diastolic myocardial dysfunction and endothelial microvascular dysfunction and significantly more often diagnosed with obstetrics complications in pregnant women with MVP and extrasystolic arrhythmia on the background of UCTD. There was a significant reduction of arrhythmias in patients with MVP and frequent ventricular (90.6\%) or mixed extrasystoles (77.8\%) under the influence of complex drug treatment with L-arginine and L-carnitine.

Conclusion: There are violations of morpho-functional parameters of the heart with the development of moderate systolic-diastolic myocardial dysfunction and endothelial microvascular dysfunction, which are triggers of complicated pregnancy and childbirth in pregnant women with MVP and extrasystolic arrhythmia on the background of UCTD in the initial state. The inclusion of L-arginine and L-carnitine led to a significant improvement in the parameters of central cardiohemodynamics and restoration of endothelial function of microvessels and pregnancy complications.
\end{abstract}

Keywords: Pregnancy; Undifferentiated Connective Tissue Dysplasia (UCTD); Mitral Valve Prolapse (MVP); Extrasystole; Systolicdiastolic and Endothelial Dysfunction; Arginine; Carnitine

\section{Introduction}

Anomalies of labor are one of the main sources of surgical delivery, obstetric and perinatal trauma, uterine bleeding. Analysis of the causes of these disorders allowed to determine the role of congenital and acquired abnormalities in the development of connective tissue (CT) in the functional state of the pregnant uterus 
[1-3]. The frequency of diagnosis of undifferentiated connective tissue dysplasia (UCTD) among women of reproductive age reaches $33.1 \%$ [4-6]. The most common of them are vegetative-vascular dystonia, structural abnormalities of the heart (mitral or aortic valve prolapse, venous varicose processes, genital prolapse, etc.), which complicate the course of pregnancy [7-9]. The prevalence of mitral valve prolapse (MVP) predominant in female patients, and its negative impact on myocardial contractility and frequent combination with cardiac arrhythmias and conduction can predict the adverse effects on pregnancy, childbirth in women and the development of the fetus [10-12].

The unity between CT defects and the formation of uteroplacental insufficiency is also proved not only due to the defeat of connective tissue fibers of elastic or mixed type arteries, but also through the development of endothelial microvascular dysfunction $[13,14]$, which causes a tendency to uterine bleeding (menorrhagia, pathological blood loss after childbirth and abortion), and in the perinatal aspect - an increased frequency of hemorrhages, in particular, cephalohematoma [15-17].

These mechanisms of development the phenotypic manifestations of CT dysplasia in pregnant women and triggering effect on ante- and perinatal complications suggest the possibility of metabolic and cytoprotective therapy on these pathological processes. The most promising and physiological metabolic drugs are L-carnitine and L-arginine. L-arginine has antihypoxic, membrane stabilizing, antioxidant and detoxifying activity, manifests as an active regulator of intermediate metabolism and supply energy processes [18,19], but its main physiological role is to regulate the level of function body organs and tissues [20,21]. L-carnitine occurs an important role in energy metabolism of myocardium due to the transfer of free fatty acids from the cytosol into mitochondria and providing bioavailability of high-energy substrate for oxidative metabolism in cardiomyocytes [22,23], which positively affects left ventricular metabolism and function [24-26]. The above facts became the basis for the study of clinical efficacy and the possibility use of arginine-carnitine complex for correction of arrhythmias on the background of systolic and endothelial dysfunction for reduce the incidence of obstetric complications in pregnants with UCTD.

\section{The Aim of the Study}

To reduce the incidence of obstetric complications associated with cardiovascular disorders by including carnitine-arginine complex for treatment of pregnant women with UCTD.

\section{Materials and Methods}

The work is based on the results of a comprehensive examination of 58 pregnants with 2 - 8 individual signs of undifferentiated connective tissue dysplasia, including MVP with and without frequent extrasystoles. The age of patients ranged from 19 to 30 (mean $21.9 \pm 2.8$ ) years. The international phenotypic scale of M. Gleshbi (1989) was used for all women to identify UCTD syndrome, and the evaluation table of LN Fomina (2000) was used to determine the clinical significance of the set of UCTD symptoms within moderate and severe dysplasia.

The study included: 1) general clinical and laboratory biochemical examination; 2) questionnaire to identify phenotypic markers of connective tissue dysplasia (CTD) and stigmas of dysembryogenesis, heredity of various diseases; 3) ECG - research in 12 standard leads; 4) HM ECG with the help of software and hardware complex «DiaCard» («Solveig», Ukraine); 5) Echocardiography - research in M-, B- and D-modes on the device «Toshiba istyle SSA-580A»; 6) Ultrasound (US) included feto- and placentometry, biophysical profile, Doppler hemodynamics in the uterus-placenta-fetus, assessment of uterine vessels for varicose transformation of uterine and internal iliac veins was performed by ultrasound scanner «Sonoskope-30» («Kranzbuhler», Germany); 7) Fetus cardiotocography was performed with a Fetalcare device (Kranzbuhler, Germany); 8) The functional state of the vascular endothelium was determined by the concentration of endothelin-1 (ET-1) in blood plasma (using the enzyme-linked immunosorbent assay «Amersham Pharmacia Biotech» and affinity chromatography columns) and the content of stable NO metabolites in reaction with Gris reagent. The amount of nitrites was calculated according to the calibration schedule [27].

For prenatal care of pregnant women with UCTD and prevention of obstetric complications associated with cardiovascular disorders in the experimental group, we used metabolic support prescribing in addition to standard protocol treatment of $4.2 \mathrm{~g}$ of L- arginine and $2.0 \mathrm{~g}$ of L-carnitine in solution for intravenous infusion once a day during 10 days. The control group included 20 pregnants with standard management of women with UCTD before childbirth. The reference indicators were takes from 14 healthy pregnant women of the same age and gestation.

Evaluation of clinical and antiarrhythmic efficacy of therapy was performed according to clinical and instrumental data after 10-15 days of treatment, repeated comprehensive examination - at 36 weeks of gestation and first day after delivery. 
Statistical processing of the obtained results was performed using the statistical software package SPSS «Statistica v18» and the program «Microsoft Exel-2013». To evaluate the data used nonparametric statistical methods - Mann-Whitney U-test to compare indicators in the two groups $(\mathrm{p}<0,05)$.

\section{Research Results and discussion}

The frequency of UCTD was determined at $29.3 \%$ by retrospective analysis of the examined pregnant women, which, in general, corresponds to the population prevalence of UCTD in Ukraine [7]. Obstetric risk factors included such constitutional features characteristic of UCTD as asthenic physique with a deficit of body weight (BP 5.57; CI 4.01-7.67; p < 0.05), body weight less than $55 \mathrm{~kg}$ before pregnancy (BP 2.4; CI 1.48-3.64; $\mathrm{p}$ $<0.05)$. Among the pregestational factors are often identified the following pathological conditions, that considerably «associated» with UCTD: mitral valve prolapse, arterial hypotension, vegetativevascular dystonia, veins varicose, caries, myopia, chronic tonsillitis, predisposition to frequent colds, bronchial diseases, biliary discinesia, pathology of the urinary organs. The reproductive history showed a high frequency of premature birth (BP 4.43; CI $1.87-10.52 ; \mathrm{p}<0.05$ ), the risk of miscarriage (BP 1.88; CI 1.073.31; $\mathrm{p}<0,05$ ), perinatal losses (BP 1.57; CI 5.13-16.58; $\mathrm{p}<0.05$ ), uterine postpartum bleeding (BP 3.05; CI 2.03-4.55; $\mathrm{p}<0.05$ ), which statistically confirms the pathological effect of UCTD on pregnancy and childbirth.

The consequences of pregnancy associated with UCTD was early preterm birth at 22-27 weeks (BP 4.9, CI 2.27-10.61, p=0.001). The duration of labour was on average shorter (respectively $286 \pm 15$ minutes and $332 \pm 21$ minutes) ( $p<0,05$ ), but in labour with UCTD there was a higher frequency of pathological preliminary period (PP) $(9.2 \%$ vs. $3,1 \%)$ and primary uterus inertia (7.7\% vs. $3.4 \%$; $<0.001$ ), whereas in the absence of UCTD - more often diagnosed with secondary uterus inertia (1.5\% and $2.8 \%$, respectively), Amniotic sac rupture was also highly associated with UCTD (BP 8.96; CI 6.72-11.96; $\mathrm{p}<0.001$ ).

79.3\% women with UCTD has natural delivery, and the high frequency of cesarean sections (20.7\%) twice highly to the control, which allows to consider persons with UCTD as a risk group for operative delivery and urgent cesarean section due to pathological labour.
Thus, the results of a retrospective study led to the conclusion that the presence of signs of UCTD is obstetric risk factor and a prerequisite for hereditary nature in the development of birth defects.

Other specific risk factor for development of pathological course of pregnancy and childbirth is presence of MVP in combination with frequent extrasystoles. Phenotypic markers of arrhythmogenesis in women with MVP should be considered: height $>180 \mathrm{~cm}$, joint hypermobility, increased skin signs of wrist and thumb, varicose veins $(\mathrm{p}<0,001)$. It was also noted that joint hypermobility, increased skin signs of wrist and thumb show positive correlations ( $r=0.19-0.25, \mathrm{p}<0.05$ ) with such characteristics of MVP as the degree of prolapse of the anterior mitral valve, the presence of myxomatous degeneration of the valves, mitral regurgitation and bicuspid prolapse, which in some way determine the severity and features of MVP in pregnant women.

There were 32 (55.2\%) with frequent ventricular arrhythmias, $18(31.0 \%)$ - with frequent supraventricular arrhythmias and 8 (13.8\%) - with combined arrhythmias: frequent ventricular and supraventricular arrhythmias in pregnants with MVP. In pregnant women with MVP and frequent ventricular arrhythmias, the daily number median of arrhythmia was 870.0, and in 1 hour search 38.4. Almost half (49.1\%) of these patients had binary and group extrasystoles (the daily number median of binary and group ventricular extrasystoles was 37.5). In patients with frequent supraventricular extrasystole, the median value of the daily amount of extrasystole was 956.0, and in 1 hour search 42.8. Episodes of sinus tachycardia were observed in $68.0 \%$ patients with frequent supraventricular arrhythmias during the day (daily number median of episodes was 2.0, maximum duration of episodes was $12 \mathrm{~s})$.

The results of the echocardiographic study analysis showed that there is a significant increase in the value of left ventricular end-systolic dimension (LVESD) and its index (ESVI) (35.1 vs. 30.8 $\mathrm{mm}$ and 18.9 vs. $16.7 \mathrm{~mm} / \mathrm{m}^{2}$, respectively, $\left.\mathrm{p}<0,05\right)$, LV posterior wall thickness (LVPWs) (9.7 vs. $8,9 \mathrm{~mm}, \mathrm{p}<0,05$ ), interventricular septum (IVS) thickness (9.8 vs. $8.8 \mathrm{~mm}, \mathrm{p}<0.001$ ), myocardial wall thickness $(0.39$ vs. $0.35, \mathrm{p}<0.05)$, right ventricular size (33.0 against $29.0 \mathrm{~mm}, \mathrm{p}<0.05$ ), left ventricular mass index (LVMI) (114.8 vs. $96.5 \mathrm{~g} / \mathrm{m}^{2}, \mathrm{p}<0.05$ ), deceleration time of early 
diastolic filling (DT) against $166 \mathrm{~ms}, \mathrm{p}<0,05)$ and isovolumetric myocardial relaxation (IVRT) (82 against $71 \mathrm{~ms}, \mathrm{p}<0,05)$, reduction of emission fraction (EF), rate of circular contraction of myocardial fibers (Vcf) (1.11 vs. $1.22 \mathrm{~m} / \mathrm{s}, \mathrm{p}<0,05)$, left atrium/ right atrium ratio (1.2 vs. $1.3, \mathrm{p}<0.05)$ in pregnant women with MVP and extrasystole, in contrast to patients without arrhythmia. Thus, the presence of extrasystole in pregnant women with MVP is associated with more pronounced disorders of intracardial hemodynamics, thus - with signs of initial structural and geometric remodeling of the walls and chambers of the heart with a tendency to dilatation of ventricles and atria, deterioration of contractile and relaxation properties of myocardium. In turn, the analysis of the valvular apparatus in pregnant women with MVP and extrasystole, compared with patients with MVP without arrhythmia, revealed a significant increase in the thickness of the mitral valve leaflets ( 1.5 vs. $1.0 \mathrm{~mm}, \mathrm{p}<0.05$ ), the frequency of registration cases of abnormal chords of the left ventricle ( 45.3 vs. $28.3 \%, p<0.05$ ) and right ventricle ( 26.2 vs. $9.4 \%, p<0.001$ ). Probably these changes in echostructural abnormalities of the heart can be considered as structural predictors of arrhythmias in patients with MVP.

To clarify this assumption, we evaluated the effectiveness of cardioprotective and antiarrhythmic effects of carnitinearginine complex in pregnant women with MVP and extrasystolic arrhythmia. In the course of the study it was found that under the influence of complex drug treatment with the inclusion of $\mathrm{L}$-arginine and L-carnitine there is a significant decrease in the frequency of arrhythmias and conduction, but patients in this group remained significantly higher sinus tachycardia. This result can be explained by an additional decrease in systolic and diastolic blood pressure due to improved microcirculation under the influence of L-arginine. However, we note that after completing the course of the proposed complex treatment (10 days) the frequency of arrhythmias in pregnant women of the researched group was significantly reduced and was lower compared to the control group $(\mathrm{P}<0,05)$. The best effect of complex treatment was obtained in the group of patients with MVP and frequent ventricular arrhythmias or with mixed extrasystolic arrhythmia. Registration of the extrasystole frequency decreased by $90.6 \%$ and $77.8 \%$, respectively after treatment, the median daily number of extrasystoles in pregnant women in these groups was 284 and for 1 hour of the study - 7.4. The antiarrhythmic effect observed only in $37.5 \%$ patients in pregnant women with supraventricular arrhythmias, which justified the additional use of nebivolol hydrochloride at a dose $2.5 \mathrm{mg}$ /day.

Echocardiographic study (Table 1) not differ statistically in the initial state of researched and control groups, but differ significantly from those in healthy people (reference values). In the initial state in pregnant women with MVP was diagnosed systolic and diastolic $\mathrm{LV}$ dysfunction, as evidenced of increasing of LV End-diastolic volume, E, A, E/A and decreasing of LV End-systolic volume, EF, IVRT and DT. Contractile left ventricular function in pregnant women with MVP and frequent extrasystoles decreased moderately by an average of $3.8 \%$. Under the influence of standard therapy up to 10 days in pregnant women of control group indicators of LVEDV, IVRT, DT, A increased insignificantly and, accordingly, such indicators of intracardiac hemodynamics as E, E/A, EF decreased. Thus, in pregnant women with MVP gradually formed signs of diastolic dysfunction, mainly relaxation type. The obtained data indicate insufficient hemodynamic efficacy of standard therapy in pregnant women with frequent extrasystoles on the background of MVP.

\begin{tabular}{|l|c|c|c|c|c|c|c|}
\hline \multicolumn{2}{|l}{$\begin{array}{l}\text { Metrics and their reference } \\
\text { values }\end{array}$} & $\begin{array}{c}\text { Before } \\
\text { treatment }\end{array}$ & $\begin{array}{c}\text { By 10 days of } \\
\text { treatment }\end{array}$ & After delivery & p1 & p2 & p3 \\
\hline \multirow{2}{*}{$\begin{array}{l}\text { LV EDV, ml } \\
(156,4 \pm 1,4)\end{array}$} & 1 & $150,5 \pm 1,3$ & $157,2 \pm 1,3^{*}$ & $163,2 \pm 1,2^{*}$ & $<0,05$ & $<0,05$ & $<0,05$ \\
\hline \multirow{2}{*}{$\begin{array}{l}\text { LV ESV, ml } \\
(81,3 \pm 3,8)\end{array}$} & 1 & $88,6 \pm 2,3$ & $82,5 \pm 2,2$ & $81,8 \pm 2,4^{*}$ & $<0,05$ & $>0,05$ & $<0,05$ \\
\hline \multirow{2}{*}{$\begin{array}{l}\text { EF, \% } \\
(61,8 \pm 0,3)\end{array}$} & 2 & $88,9 \pm 2,1$ & $87,4 \pm 2,2$ & $89,2 \pm 2,4$ & $>0,05$ & $>0,05$ & $>0,05$ \\
\hline \multirow{2}{*}{ IVRT,Mc $(88,5 \pm 2,1)$} & 2 & $58,9 \pm 0,4$ & $57,3 \pm 0,4$ & $58,2 \pm 0,4$ & $>0,05$ & $>0,05$ & $>0,05$ \\
\cline { 2 - 8 } & 2 & $85,7 \pm 1,4$ & $84,3 \pm 1,4$ & $86,3 \pm 2,1$ & $>0,05$ & $>0,05$ & $>0,05$ \\
\hline
\end{tabular}


Correction of Cardio-hemodynamics and Endothelial Function Disorders in Pregnant Women with Connective Tissue Dysplasia

\begin{tabular}{|c|c|c|c|c|c|c|c|}
\hline \multirow{2}{*}{$\begin{array}{l}\text { DT,мс } \\
(196,4 \pm 4,7)\end{array}$} & 1 & $180,8 \pm 5,6$ & $186,4 \pm 5,3$ & $215,6 \pm 4,1^{*}$ & $>0,05$ & $<0,05$ & $<0,05$ \\
\hline & 2 & $179,5 \pm 4,4$ & $180,6 \pm 4,6$ & $184,6 \pm 4,2$ & $>0,05$ & $>0,05$ & $>0,05$ \\
\hline \multirow{2}{*}{$\begin{array}{l}\text { Е, см/сек }(45,67 \\
\pm 1,7)\end{array}$} & 1 & $54,4 \pm 1,6$ & $48,1 \pm 1,2 *$ & $45,8 \pm 1,7^{*}$ & $<0,05$ & $>0,05$ & $<0,05$ \\
\hline & 2 & $53,2 \pm 1,3$ & $52,7 \pm 1,3$ & $49,9 \pm 1,4$ & $>0,05$ & $>0,05$ & $>0,05$ \\
\hline \multirow{2}{*}{$\begin{array}{l}\text { А, см/сек }(36,8 \pm \\
1,1)\end{array}$} & 1 & $41,5 \pm 1,2$ & $37,4 \pm 1,2 *$ & $37,1 \pm 1,1^{*}$ & $<0,05$ & $>0,05$ & $<0,05$ \\
\hline & 2 & $40,8 \pm 1,3$ & $41,7 \pm 1,1$ & $42,7 \pm 1,2$ & $>0,05$ & $>0,05$ & $>0,05$ \\
\hline \multirow{2}{*}{$\begin{array}{l}\mathrm{E} / \mathrm{A} \\
(1,23 \pm 0,04)\end{array}$} & 1 & $1,31 \pm 0,05$ & $1,29 \pm 0,03$ & $1,19 \pm 0,02^{*}$ & $>0,05$ & $<0,05$ & $<0,05$ \\
\hline & 2 & $1,29 \pm 0,05$ & $1,30 \pm 0,06$ & $1,08 \pm 0,05$ & $>0,05$ & $<0,05$ & $<0,05$ \\
\hline \multicolumn{8}{|c|}{$\begin{array}{l}\text { Notes: } 1.1 .2 \text { - respectively indicators in pregnant research }(\mathrm{n}=58) \text { and control }(\mathrm{n}=20) \text { groups; } 2 . \mathrm{p} 1, \mathrm{p} 2, \mathrm{p} 3 \\
\text { - accordingly, the reliability of the difference between the parameters in pregnant women before and after } 10 \\
\text { days of treatment, after } 10 \text { and after delivery and between patients before treatment and after childbirth; } 3 .{ }^{*} \\
\text { indicators are reliably different from similar groups of control in pregnant women. }\end{array}$} \\
\hline
\end{tabular}

Table 1: Dynamics of echocardiogram parameters under the influence of the proposed treatment program in pregnant women with MVP and extrasystole $(\mathrm{M} \pm \mathrm{m})$.

In pregnant women of researshed group, who reseaved arginine-carnitine complex in addition to standard therapy treatment, re-examination showed a significant increase of EF and IVRT, as well as a decrease of LVEDV and E and A. Changes in other hemodynamic parameters were insignificant compared to initial data, however, there was a tendency to reduce the manifestations of post-extrasystolic remodeling of the heart. At the same time, the preservation of the achieved therapeutic effect in the postpartum period was noted. Thus, in women in labor after complex therapy significantly increased, the rate of IVRT, DT, LVEDV and EF and decreased LVESV, E, A, E/A compared to baseline. Thus, the addition of arginine and carnitine to the standard medication of pregnant women helped to reduce the size of the left ventricles, manifestations of post-arrhythmic remodeling of the left ventricle, which ultimately manifested a significant increase in EF (average $3.5 \%$ ), improved myocardial contractility and reduced dysfunction.
A promising task was also to investigate the therapeutic efficacy of the proposed comprehensive treatment of endothelial microvascular dysfunction. It was found that in the initial state, the indicators of the functional state of the endothelium in all pregnant women with MVP and frequent extrasystoles did not differ significantly, but were significantly impaired in comparison with the reference data (Table 2). During treatment in patients of control group, who underwent standard protocol drug support of pregnancy, the level of endothelin-1 was significantly reduced, and did not reach the level of healthy pregnant women $(\mathrm{P}<0.05)$. At the same time, in pregnant women of researched group, who in addition to the complex treatment included a course of therapy with L-arginine and L-carnitine during 10 days, the activity of ET-1 decreased by $42.7 \%(\mathrm{P}<0.05)$. Thereby, this treatment in pregnant women with MVP and frequent extrasystoles contributed to a rapid decrease in the activity of endothelin-1 in blood plasma.

\begin{tabular}{|l|c|c|c|c|c|}
\hline \multicolumn{2}{|l}{$\begin{array}{l}\text { Metrics and their } \\
\text { reference values }\end{array}$} & \begin{tabular}{l} 
before treatment \\
\hline \multirow{3}{*}{ Endoteline-1, ng/ml }
\end{tabular} & $\begin{array}{c}\text { By 10 days of } \\
\text { treatment }\end{array}$ & p1 & p2 \\
\cline { 2 - 6 } & 2 & $0,46 \pm 0,03$ & $0,47 \pm 0,04$ & $>0,05$ & $>0,05$ \\
\cline { 2 - 6 } & 3 & $0,89 \pm 0,03$ & $0,51 \pm 0,05$ & $<0,05$ & $>0,05$ \\
\hline \multirow{3}{*}{$\mathrm{NO}_{2}, \mathrm{mkmol} / \mathrm{l}$} & 1 & $11,46 \pm 0,34$ & $11,56 \pm 0,43$ & $>0,05$ & $>0,05$ \\
\hline & 2 & $6,89 \pm 0,42$ & $10,85 \pm 0,39$ & $<0,05$ & $>0,05$ \\
\hline \multirow{3}{*}{$\mathrm{NO}_{3}, \mathrm{mkmol} / \mathrm{l}$} & 3 & $6,68 \pm 0,43$ & $7,71 \pm 0,32$ & $>0,05$ & $<0,05$ \\
\cline { 2 - 6 } & 1 & $25,46 \pm 0,53$ & $25,58 \pm 0,63$ & $>0,05$ & $>0,05$ \\
\cline { 2 - 6 } & 2 & $16,83 \pm 0,46$ & $21,87 \pm 0,51$ & $<0,05$ & $<0,05$ \\
\cline { 2 - 6 } & 3 & $16,87 \pm 0,43$ & $19,32 \pm 0,42$ & $<0,05$ & $<0,05$ \\
\hline
\end{tabular}

Citation: Svitlana Mykolaivna Heryak., et al. "Correction of Cardio-hemodynamics and Endothelial Function Disorders in Pregnant Women with Connective Tissue Dysplasia". Acta Scientific Women's Health 4.2 (2022): 32-39. 


\begin{tabular}{|c|c|c|c|c|c|}
\hline \multirow{3}{*}{$\mathrm{NO \varepsilon}, \mathrm{mkmol} / \mathrm{l}$} & 1 & $35,92 \pm 0,37$ & $36,04 \pm 0,47$ & $>0,05$ & $>0,05$ \\
\hline & 2 & $23,75 \pm 0,42$ & $33,74 \pm 0,52$ & $<0,05$ & $>0,05$ \\
\hline & 3 & $23,37 \pm 0,42$ & $27,65 \pm 0,52$ & $<0,05$ & $<0,05$ \\
\hline \multicolumn{6}{|c|}{$\begin{array}{l}\text { Notes: } 1.1,2,3 \text { - accordingly, indicators in reference, researched and control group; } 2 . \mathrm{p} 1, \mathrm{p} 2 \text { - accordingly, } \\
\text { reliability of the difference between parameters before and after treatment and after treatment and reference } \\
\text { indicators; } 3 \text {. The underlined indicators are reliably different from the corresponding group of healthy } \\
\text { individuals. }\end{array}$} \\
\hline
\end{tabular}

Table 2: Dynamics of endothelial microvascular function in pregnant women with MVP and extrasystole under the influence of the proposed treatment program $(\mathrm{M} \pm \mathrm{m})$.

Simultaneously with changes of endothelin-1 activity in pregnant women with MVP and extrasystole, there was a decrease in the level of nitric oxide metabolites, which can indicate a pronounced violation of microcirculation in these patients. Thus, the concentration of nitrates and nitrites in the initial state in pregnant women of both groups decreased 1.7 times, and their total content in blood plasma decreased by $34.3 \%$ compared with the values of reference indicators in pregnant women. Generally accepted protocol of pregnancy in the presence of UCTD stigmas did not provide complete restoration of vascular endothelial function in the control group of pregnant women with MVP and extrasystolic arrhythmia. Total concentration of nitric oxide metabolites in plasma was $23.5 \%$ lower $(\mathrm{P}<0.05)$. At the same time, the use of complex drug therapy with the inclusion of arginine-carnitine complex in patients of researched group significantly affected concentration of nitrites and nitrates in blood plasma, their level increased significantly after 10 days of treatment and reached the reference norm.

Thus, the results of a comprehensive study of clinical and functional status of pregnant women with phenotypic signs of UCTD, MVP and extrasystolic arrhythmia allow us to conclude that in most cases this pathology occurred on the background of moderate heart failure and significant endothelial dysfunction of bed vascular situation in pregnant women. There was no significant reduction in the frequency of arrhythmias, improvement of inotropic capacity of the myocardium and restoration of endothelial function of microvascular tissues after standard medical support in pregnant women of control group. Supraventricular and ventricular arrhythmias remained resistant to standard treatment, which justified the use of additional antiarrhythmic therapy with nebivolol hydrochloride. At the same time, pregnant women with MVP and extrasystolic arrhythmia of researched group, who additionally included L-arginine and L-carnitine in complex treatment showed a significant decrease of arrhythmias, improved myocardial contractility and restoration of endothelial microvascular function.

In our opinion positive impact of the proposed treatment on the inotropic function of heart and a significant reduction in the frequency and severity of extrasystolic arrhythmias are achieved due to cardiometabolic effects of L-carnitine, that matters its important role in myocardial energy metabolism by transfer of free fatty acids from cytosol to mitochondria and thus provides bioavailability of a high-energy substrate for oxidative metabolism in the cardiomyocyte $[22,25]$. In addition, by facilitating the oxidation of long-chain fatty acids and modulating the ratio of CoA to $\mathrm{CoA}-\mathrm{SH}$, the compound is involved in the binding of acyl residues in peroxisomes and mitochondria and has a positive effect on amino acid metabolism, assimilating the array of free radicals and cells prevents the accumulation in the cytoplasm of cardiomyocytes of fatty acid esters, which can lead to fatal ventricular arrhythmias $[21,23]$.

We also found that such comprehensive treatment in pregnant women with MVP and extrasystolic arrhythmia on the background of UCTD contributed to rapid and complete recovery of studied indicators of endothelial function of microvessels. The obtained result can be explained by the use of $\mathrm{L}$-arginine as the main substrate for the synthesis of nitric oxide. Many studies showed that this effect of L-arginine is achieved due to its antihypoxic, antioxidant and membrane stabilizing activity, but its main physiological role is to regulate the functional state of microvessels and ensure the appropriate level of microcirculation of organs and tissues $[28,29]$. 
In general, due to the complex use of combination drug therapy with the inclusion of L-arginine and L-carnitine in pregnant women with MVP and extrasystolic arrhythmia on the background of UCTD, a significant improvement in the parameters of central cardiohemodynamics and restoration of endothelial function was reduced.

\section{Conclusions}

- There are violations of morpho-functional parameters of the heart with the development of moderate systolic-diastolic myocardial dysfunction and endothelial microvascular dysfunction in pregnant women with MVP and extrasystolic arrhythmia on the background of UCTD in the initial state.

- Morpho-functional changes of heart parameters and metabolic disorders in comorbid pregnant women with MVP and extrasystolic arrhythmia on the background of UCTD led to the development of complicated pregnancy and childbirth. They were significantly more often diagnosed with miscarriage (isthmic-cervical insufficiency, miscarriage, premature birth), late preeclampsia, placental dysfunction, premature rupture of membranes, anomalies of placement and attachment of the placenta, abnormalities in labor, postpartum trauma.

- The use of complex drug therapy with L-arginine and L-carnitine in pregnant women with MVP and extrasystolic arrhythmia on the background of UCTD led to a significant improvement in central cardiohemodynamics and restoration of endothelial function of microvascules, accompanied by a change in essence and development.

\section{Bibliography}

1. Faiz SA., et al. "Pregnancy and valvular heart disease". Saudi Medical Journal 24.10 (2016): 1098-1101.

2. Gazazyan MG. "Features of the course of pregnancy and childbirth in patients with connective tissue dysplasia". Obstetrics and Gynecology 1 (2017): 121-126.

3. Jana N., et al. "Pregnancy in association with mitral valve prolapse". Asia-Oceania Journal of Obstetrics and Gynaecology 19.1 (1993): 61-65.

4. Kildiyarova RR and Uglova DF. "Dysplasia of connective tissue as a constitutional basis of congenital heart defects in women and their newborn children". Archives of Internal Medicine 4 (2015): 38-42.
5. Makarchuk OM., et al. "Undifferentiated connective tissue dysplasia as a factor of probable gestational complications". Genetics / Obstetrics and Gynecology 2 (2015): 18-19.

6. Yashchuk AG., et al. "Functioning of the hemostasis system in pregnant women against the background of undifferentiated connective tissue dysplasia". Practice Medicine 1.93 (2016): 37-40.

7. Siromakha SO., et al. "Marfan syndrome and pregnancy". Actual Problems of Pediatrics, Obstetrics and Gynecology 1 (2020): 94-101.

8. Ben Salkha M and Repina NB. "Clinical diagnosis of undifferentiated connective tissue dysplasia”. I.P.Pavlov Russian Medical Biological Herald 24.4 (2016): 164-172.

9. Pavlenko M. "Isthmic-cervical insufficiency: description, causes, treatment" (2018).

10. Hameed A., et al. "The effect of valvular heart disease on maternal and fetal outcome of pregnancy". Journal of the American College of Cardiology 37.3 (2015): 893-899.

11. Smirnova TL., et al. "Features of the course of pregnancy and childbirth in women with the syndrome of undifferentiated connective tissue dysplasia". Practice Medicine 16.6 (2018): 39-44.

12. Lukina TS., et al. "Management of pregnant women with undifferentiated connective tissue dysplasia". 4 (2015): 2-5.

13. Heryak SM., et al. "Electrolyte disorders as a predictor of obstetric and perinatal complications in pregnant women with mitral valve prolapse". Coll. of Sci. Works of Obstet. Gynecol. of Ukraine. (2019): 11-19.

14. Saryyeva OP., et al. "Pathomorphology of extra-placental membranes with their premature rupture and undifferentiated connective tissue dysplasia in women". Archives of Pathology 81.4 (2019): 26-32.

15. Tetelyutina FK., et al. "Indicators of biopolymers of connective tissue dysplasia in the assessment of placental insufficiency". Vyatka Medical Bulletin 2.58 (2018): 27-31.

16. Chia YT., et al. "Pregnancy outcome and mitral valve prolapse". Asia-Oceania Journal of Obstetrics and Gynaecology 20.4 (1994): 383-388. 
17. Nanna M and Stergiopoulos K. "Pregnancy complicated by valvular heart disease: an update". Journal of the American Heart Association 3.3 (2014): 76-80.

18. Kazachkova EA. "Syndrome of dysplasia of the connective tissue of the heart and pregnancy". Obstetrics and Gynecology 1 (2017): 127-132.

19. Sitnikova OG., et al. "Free radical oxidation of lipids and antioxidant activity in pregnant women with undifferentiated connective tissue dysplasia in premature pregnancy". Tavrida Med. Biol. Bull. 1.69 (2015): 120-122.

20. Kononenko IS. "Polymorphisms of the genes of the transforming growth factor $\beta 1$ and matrix metalloproteinase 9 as molecular genetic predictors of the formation of isthmic-cervical insufficiency in patients with undifferentiated connective tissue dysplasia". Bulletin VSMU 19.3 (2020): 50-58.

21. Dobrokhotova YuE and Borovkova YeI. "Pregravid preparation and management of pregnancy in patients with connective tissue dysplasia". Gynecology 19.5 (2017): 44-49.

22. Ilina YuI and Chikisheva AA. "Features of the course of pregnancy in patients with connective tissue dysplasia". RMJ. Mother and Child 3 (2020): 182-188.

23. Dobryanska VYu., et al. "Predictors of extrasistic arrythmia and its treatment in pregnant military valve prolapses". Advances in Clinical and Experimental Medicine 2 (2018): 48-52.

24. Lichodziejewska B., et al. "Clinical symptoms of mitral valve prolapse are related to hypomagnesaemia and attenuated by magnesium supplementation". American Journal of Cardiology 79.6 (2017): 768-772.

25. Artal R., et al. "Transient ischemic attack: a complication of mitral valve prolapse in pregnancy". Obstetrics and Gynecology 71.6 (2016): 1028-1030.

26. Kucharczyk-Petryka E., et al. "Mitral valve prolapse at pregnancy: is it a real clinical problem?" Polish Archives of Internal Medicine 114.5 (2016): 1084-1088.

27. Smolnova TYu. "Indications for the choice of drug therapy in patients with connective tissue dysplasia in obstetrics and gynecology". Proceedings of the first All-Russian conf. Topical issues of internal pathology. Connective tissue dysplasia (2015).

28. Kondakov IL and Yakovenko AF. "Methods of morpho-functional study of endothelial status. Methodical recommendations". Kharkiv (2000): 18.
29. Selyuk MM., et al. "Selection of the optimal combination of metabolic drugs for the treatment of patients with cardiovascular pathology". Family Medicine 2.70 (2017): 60-64.

30. Shved MI and Pelyo M. "Influence of cardioprotective metabolic therapy on the occurrence of rhythm and conduction disorders in patients with myocardial infarction with metabolic syndrome". Arithmology 2.26 (2018): 53-55.

\section{Assets from publication with us}

- Prompt Acknowledgement after receiving the article

- Thorough Double blinded peer review

- Rapid Publication

- Issue of Publication Certificate

- High visibility of your Published work

Website: www.actascientific.com/

Submit Article: www.actascientific.com/submission.php

Email us: editor@actascientific.com

Contact us: +919182824667 